\title{
Experimental measurement of bending stiffness of phospho- lipid vesicles by non-invasive method
}

\author{
Penič ${ }^{1}{ }^{1 *}$, Kralj-Iglič $V^{2}$, Iglič $A^{1}$ \\ 1. Laboratory of Physics, Faculty of Electrical Engineering, University of Ljubljana, Ljubljana, Slovenia \\ 2 Laboratory of Clinical Biophysics, Faculty of Health Sciences, University of Ljubljana, Ljubljana, Slovenia \\ * Correspondence: Samo Penič; samo.penic@fe.uni-lj.si
}

Citation: Penič S, Kralj-Iglič V, Iglič

A. Experimental measurement of bending stiffness of phospho-lipid vesicles by non-invasive method. Proceedings of Socratic Lectures. 2021; 6: 115-123.

https://doi.org/10.55295/PSL.2021.D. 015

Publisher's Note: UL ZF stays neutral with regard to jurisdictional claims in published maps and institutional affiliations.

Copyright: (C) 2021 by the authors. Submitted for possible open access publication under the terms and conditions of the Creative Commons Attribution (CC BY) license (https://creativecommons.org/licenses/b $\mathrm{y} / 4.0 /)$.

\begin{abstract}
:
Biological membranes, thin barriers between compartments, can fluctuate due to their interacting mechanical properties. They behave as two-dimensional liquids and are so soft that collision of molecules in the surrounding media can cause undulations of the shape. Flickering of red blood cells was already recorded in the late 19th century by Browicz using the light microscope. Today improved contrast images can be recorded directly on computer with phase-contrast microscopy with attached cameras allowing non-invasive spectral analysis of those thermal fluctuations of biological membranes. The measurement setup must provide means for sharp and focused image acquisition for subsequent numerical determination of elasticity parameters (bending stiffness of bending elasticity). Microscope cameras have rather long integration times, resulting in blurry images, since high speed motion of the membrane is lost due to long opening of the shutter. The adaptation of phase-contrast microscope with stroboscopic illumination will be presented to achieve better resolution of acquired images, allowing higher spectral nodes to be used in the analysis of bending stiffness. Other methods of determining bending stiffness will be briefly presented to provide an overview of experimental determination of mechanical properties of phospholipid membranes.
\end{abstract}

Keywords: Phospholipid bilayer properties; Vesicle characterization; Thermal fluctuations; Phase contrast microscopy; Monte Carlo simulations 


\section{Introduction}

Biological cells are the building blocks of all life. They are very diverse in its capabilities and shape, however the basic structural elements are the same as well as its chemical compositions (Boal 2012, Phillips 2013). Cell membranes enclose the cell and its compartments, while networks of filaments, if present, help to maintain the shape of the cell. The mechanical properties of the cell elements can be much different from those of objects familiar in everyday life. In this article, we will present methods of determining the bending stiffness measurements of a lipid bilayer, that is a main building block of the cell membrane. Lipid bilayer consists of lipid molecules (Figure 1) and it is very soft. It shows very small resistance to bending, so that even thermal fluctuations at room temperature can generate gentle undulations of the membrane. To simplify the cell membrane and to analyse only the bilayer, the measurement can be done on synthetically produced giant unilamellar vesicles (GUVs). The influence of other constituents on mechanical properties can then be controllably investigated. The diameter of GUVs is in order of few microns to $200 \mu \mathrm{m}$. GUVs thus present ideal structures for investigation under optical microscope.
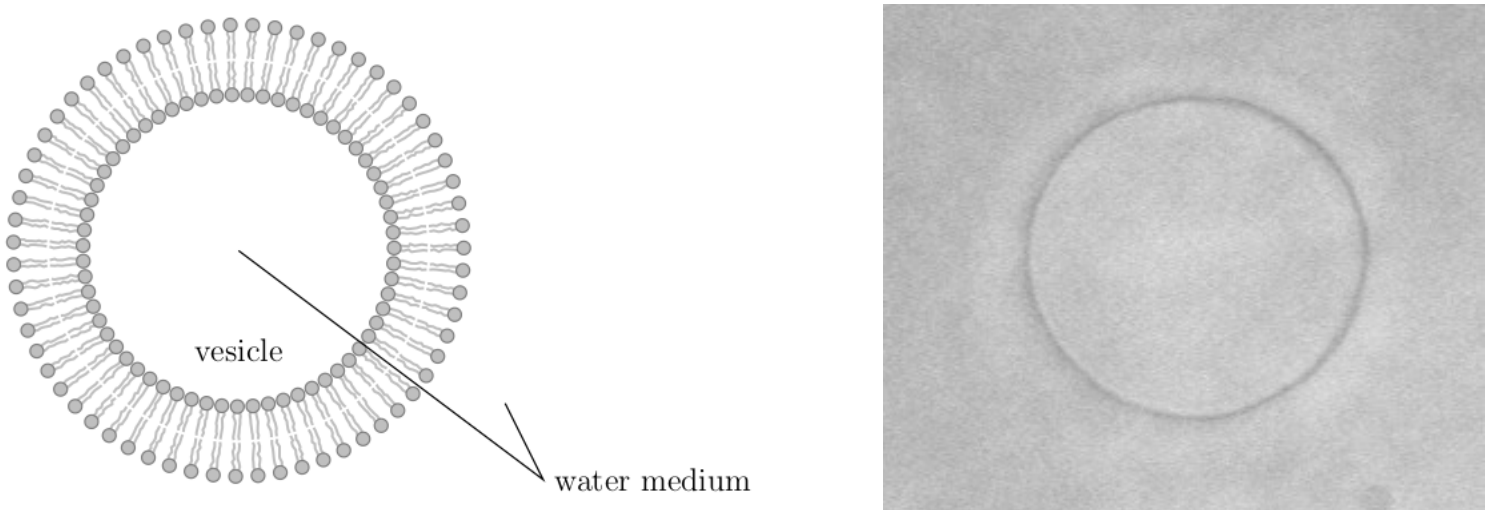

Figure 1. On the left panel, there is a schematic representation of phospholipid bilayer forming a vesicle (membrane). The right panel shows an image acquired by the digital camera attached to the viewport of phase contrast optical microscope. Phase contrast microscopy greatly emphasizes the membrane.

The study of physical properties of the cell membranes is highly important for better understanding of the working of the cell. Many studies deals with basic properties of the phospholipid membranes including the measurement of bending stiffness and the external influences or structural changes to the stiffness of the membrane (as it was for example reported in Mohandas et al. (1983), Evans and Leung (1984), Song and Waugh (1993), Zhou and Raphael (2005).

The methods for measuring the bilayer bending stiffness belong to two groups. Microscopic methods provide structural data at the molecular level, yet numerical modelling or molecular dynamics simulations are required to relate these data to macroscopic properties. Macroscopic methods offer more direct measurements on membrane response to external perturbations, but contact methods which are a common subgroup of macroscopic methods are mostly invasive, which may drive the specimen out of its natural state (Lee et al. 2001). In the following section, some of the most frequently used methods will be described, where the main focus will be on spectral analysis of thermal shape fluctuations. 
Optical macroscopic methods, such as spectral analysis of shape fluctuations do not impose external influence on the measured specimen. However, using purely optical macroscopic methods, the measurement precision is limited with optical imaging resolution, therefore many research is focused mainly on GUVs (Menger and Keiper, 1998). Furthermore, the properties of imaging sensor decrease the sharpness of the image due to fast movement of the membrane and due to a relatively long time during which the camera has its shutter open and collects light. Therefore, using fast cameras can be beneficial to improving the sharpness of the acquired image. Another approach with stroboscopic illumination was proposed by Genova et al. (2009). In this contribution, the procedure of adapting phase-contrast microscope for stroboscopic illumination will be presented from the engineering point of view.

\section{Brief overview of the methods}

Numerous methods are available to determine bending stiffness of the membrane, with their virtues and drawbacks. Taking into the account the temporal and dimensional resolution of the method, one may find that some method may be more suitable for a particular kind of membranes, but the other may be better for the others. A major drawback of some methods is that they deform the measured specimen thus changing its mechanical or other properties that may be relevant to the study. For example, fluid flow imposes mechanical loading to the membrane that can then change the structure of actin cytoskeleton of the cell (Cucina et al., 1995, Pavalko et al., 1998). Furthermore, localized mechanical stress on the membrane can be transmitted over the whole cell by means of cytoskeleton propagation (Maniotis et al., 1997). Here, the various commonly used methods will be briefly presented, however, the list of methods is far from being complete.

Atomic force microscopy (AFM) is used for characterization of materials and can be used as a method to determine bending stiffness of the phospholipid membranes (Jaasma et al., 2006, Lin et al., 2007). Observation of the deflection of the AFM cantilever beam is used to determine the "softness" of the material. The mathematical models that connect deformation and the Young's modulus and bending stiffness is based on Hertz indentation model (Lin et al., 2007). AFM method is fast (in order of milliseconds) and can be used for cells and vesicles with small radii down to $1 \mathrm{~nm}$ (Morshed et al. 2020). The method does not impose a large deforming force on the vesicle, however, the sample must be immobilized on substrate for AFM probing.

On same time and dimension scale, optical tweezers and magnetic tweezers are used. Optical tweezers (or optical trapping) use highly focused laser beam to trap and manipulate microscopic neutral dielectric particles. When particles are small compared with the laser wavelength, they behave as dipoles subjected to gradient forces due to photon scattering that is the consequence of different dielectric properties of materials. The scattering force is proportional to the intensity of the light and acts in the direction of incident light propagation (Nussenzveig 2018). The magnetic tweezers do not exert the heating stress due to strong light sources to the sample, but the sample should be treated with small ferromagnetic particles of iron oxide. The particles are usually in the micrometer range and coated with fibronectin to be able to attach to the integrin receptor on the cell (Morshed et al., 2020). The cell with ferromagnetic attachments is then exposed to external magnetic field which acts on the attachments with magnetic force. The magnetic force is then linked to bending stiffness (Fabry et al. 1999).

Electrodeformation is a method that can be used on cell membranes or vesicles on a larger scale, usually in the micrometer range (Jubery et al. 2014). Electric fields provide an alternative way of micromanipulation of vesicles. Compared to previously described micromanipulation methods, the electric field deformation is usually much cheaper while minimizing the risk of contamination the samples as for example in AFM. The most 
straightforward approach is to use direct current or DC pulses. When an electric pulse is applied to a vesicle, the vesicle deforms in relation to its mechanical properties. At the end of the pulse, the vesicle returns back to its initial shape. In the absence of excess area, relaxation depends on the properties of the membrane - stretching, viscosity and bending rigidity (Piontek et al. 2021).

Micropipetting is the last method in the brief presentation of methods used in determination of the bending stiffness of the vesicle membranes. With this method we are limited by the optical resolution of the microscope and by the ability of the operator to perform the procedure. The membrane is sucked into the glass cylinder of known inner diameter with predetermined pressure. From the length and radius of the membrane sucked into the pipette tube, the bending stiffness is calculated (Shao and Hochmuth 1996). This method is the simplest in terms of the required apparatus, but it deforms the specimen by a large extent.

In contrast to the forced mechanical deformation, the method based on thermal fluctuations is non-invasive. The method of thermal fluctuation that described in detail in the continuation, has a distinct advantage of not loading the measured specimen and thus preserves its original properties.

\subsection{Method of thermal fluctuations}

The flickering of the red blood cells, which is a consequence of the fluctuations of their membrane was observed already in 1890 by means of the bright-field optical microscope (Browicz, 1890). Thermal fluctuations of bilayer membranes of vesicles occur due to Brownian motion of the water molecules, colliding with the membrane of the vesicle, causing a localized displacement. As stated in (Schneider et al., 1984), the magnitude of the bending stiffness could be theoretically deduced by measuring the shape of the vesicle as a function of the excess of the hydrostatic pressure of water exterior over the vesicle interior, however the pressure excess is too small to measure (Schneider et al., 1984). Due to small curvature modulus, there are many thermally accessible shapes having the same area and volume that are within $k T$ (where $k=1.38 \cdot 10^{-23} \mathrm{~m}^{2} \mathrm{~kg} \mathrm{~s}^{-2} \mathrm{~K}^{-1}$ is the Boltzmann constant and $T$ the absolute temperature) of the equilibrium configuration of the flaccid vesicle. For this reasons, the shapes of a thin-walled unilamellar vesicle are observed to fluctuate.

The basic principles of spectral analysis of shape fluctuations are described in (Méléard et al., 1998). The sample vesicle equatorial cross section is focused under the microscope. For measurement of thermal fluctuations of phospholipid bilayer, the phase contrast or fluorescent microscopy is commonly used, as they emphasize the membrane outline. The series of still images taken from the experiment is analysed, to numerically evaluate the shape of the vesicle membrane (Genova et al., 2006, Fošnarič et al., 2013, Penič et al., 2015). The shapes are then developed into series of spherical harmonics functions and analzyed by a method proposed by Milner and Safran (1987). The time average of coefficients of spherical harmonics up to maximal possible mode are used to determine $\left(K_{c}\right)$

$$
\left\langle U_{l}^{m}(t)^{2}\right\rangle=\frac{k_{B} T}{K_{f}} \frac{1}{(l-1)(l+2)(\bar{\sigma}+l(l+1))} .
$$

Besides the bending stiffness, this equation has a free parameter $\bar{\sigma}$ which corresponds to the average stretching energy of the vesicle and is estimated to be near zero for perfectly fluid membranes.

The fluctuation of the synthetically prepared GUVs is therefore measured by means of merely observing the behaviour of the phospholipid bilayer in its environment without any interaction with the vesicle itself. The measurement is fairly slow in comparison 
with other methods presented in this work, as the measurement of a single vesicle takes 2-5 minutes. The reason for slow measurement process lays in the fact that the lowest modes of fluctuations are slow to dissipate. For analysis we require a series of independent shapes, thus we are required to take a still image every second in average.

\section{Results}

\subsection{Optical phase-contrast microscope adaptation}

Optical phase-contrast microscope is frequently used as research tool for observing thin phospholipid layers due to contrast enhancement of two optically different media (e.g. water and phospholipid mixture). The optical path of the microscope can be switched between ocular and camera output ports. By attaching CCD or newer CMOS camera sensor to the camera port, image can be acquired electronically and transported to computer as video signal or via common USB computer port.

The microscope modification for stroboscopic illumination was already implemented and described in (Genova et al., 2009, Genova and Pavlič, 2012). According to the distributor of Nikon microscopes, there is no light source splitter available for Nikon Eclipse TE2000-S phase-contrast microscope (that is used for research in our laboratory) on the market. The adaptation of the microscope to accept two light sources was done by means of additional aluminium wall chamber with retractable mirror to enable the additional light source port.

With the retractable mirror the experimenter can choose between the "constant intensity" halogen lamp light source or pulsing, stroboscopic flash light for sharper image registration for measurement of bending stiffness. The mirror is fixed on a hinge that can rotate about its axis and is controlled by a handle on top of the splitter fixture. The existing halogen lamp is fixed on the back of the chamber and the stroboscope light source is attached at the side with rubber spacers to reduce mechanical movement (shaking) of the microscope due to the flash tube discharge.

High intensity flash illumination, combined with CCD camera is cheap replacement for high sensitivity, high-speed camera, to register sharp images of vesicles. Most of the integration time of the camera the sample with the vesicle is in darkness, only a short burst of strong light is made during that time by the flash lamp. This produces sharp images of the vesicle in each frame and results in decreased error of determination of bending stiffness (Genova et al. 2006).

The Hamamatsu L7684 is a xenon flash lamp for industrial applications. It generates output power of $60 \mathrm{~W}$ in spectral range $240 \mathrm{~nm}$ to $2000 \mathrm{~nm}$. It has a built-in reflective mirror, which focus all light made by arc of size $3 \mathrm{~mm}$ towards the borosilicate glass opening in the aluminium case. It can be triggered with voltages from $5-10 \mathrm{kV}$ and can be operated under maximal repetition rate of $60 \mathrm{~Hz}$. To achieve full power flashes an additional capacitor box Hamamatsu E7289 is connected to the xenon Flash Lamp power supply Hamamatsu C6096. High energy flashes also results in some energy losses in the lamp, therefore the cooling jacket Hamamatsu E6611 with the brushless DC motor fan is needed to keep the lamp at the operating temperature. The cooling jacket provides at the same time the socket into which the xenon flash lamp is inserted. It provides mechanical stability and allows mounting to the adapted light chamber of the microscope.

The controller that synchronizes camera with the flash light has been made using the microcontroller ATmega16, produced by Microchip. The choice of the microcontroller is not critical, as the signals timings are well below $\mathrm{kHz}$ range. The schematics of microcontroller and with main parts of the trigger system is shown in Figure 2. The microcontroller clock is generated with the $7,3728 \mathrm{MHz}$ quartz crystal, allowing synchronization with standard UART speed. The connection with the computer for controlling the operation of the controller is made through serial connection with the FT232 adapter to enable USB connection. The speed of communication is 9600 baud 8N1. 


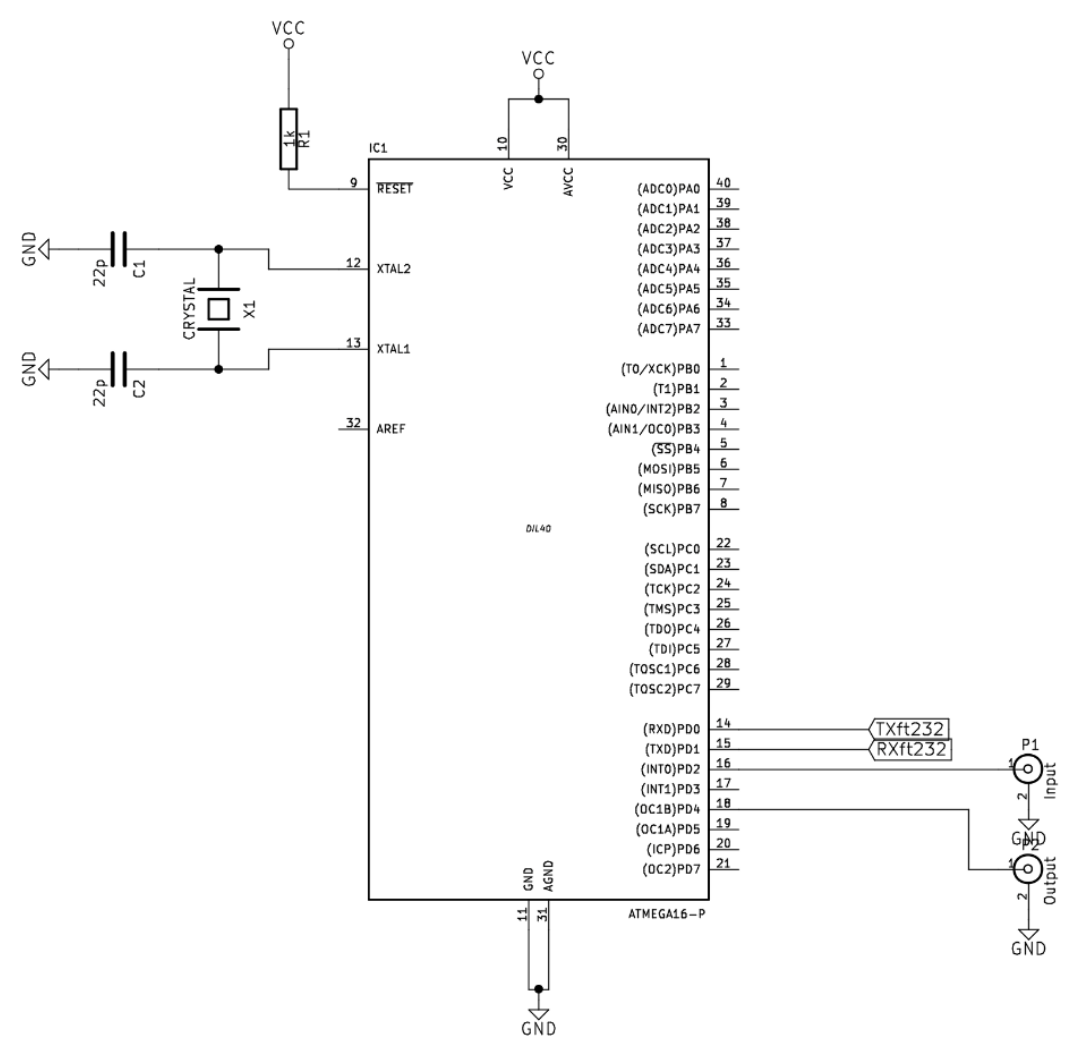

Figure 2. The electric schematics of the flash light controller using ATmega16 microcontroller. Only the most important connections are shown. At the bottom right there are input and output connections, where input presents trigger information from camera and output is trigger command signal to flash light source. The TX and RX pins are connected through RS232 to USB adapter to computer. Top left corner shows just components that are absolutely required for microcontroller to operate. By uploading the firmware to the microcontroller, the functionality can be altered, which adds to the flexibility of this design.

When the controller is switched on, the trigger is armed and waits for the camera sync pulses that generates lamp pulses for flash discharge. There is a possibility to turn on or off the trigger by sending single letter commands. This improves the automation of the measurement process. The microcontroller control of the triggering is flexible also because it is possible to programmatically set the time of the flash discharge within the integration frame of the camera if that is necessary, due to the possible camera integration start time jitter. The controller is connected to the computer via USB cable form which it also draws power.

The stroboscopic light was attached to microscope light port. There was mechanical adaptation of the microscope light port to gain additional light plug with mirror selector. The blueprint for the light mixing chamber is presented in Figure 3. On top of the chamber, stroboscopic light was mounted via 3D printed adaptor from white ABS plastics with shake reducing rubber spacer (see left panel of Figure 4 for exploded view of the final apparatus). The original halogen light source was mounted at the back of the mixing chamber. The final arrangement is shown on the photo in right panel of Figure 4. The detailed procedure of manufacturing the adaptor and light mixing chamber with two light ports is described in (Klanjšček and Penič, 2021). 


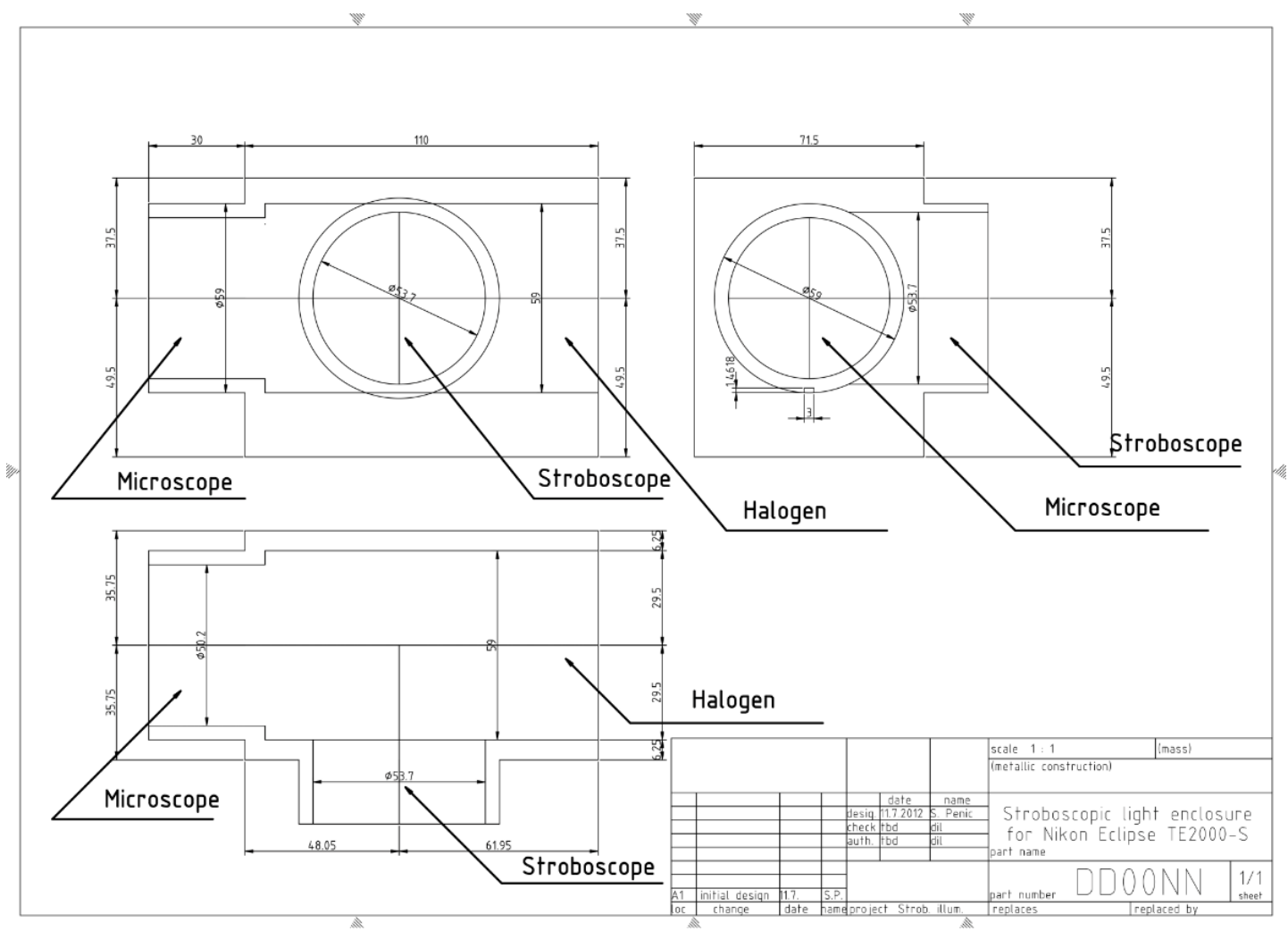

Figure 3. The blueprint for aluminium casing of light mixer with two input light ports and one output light conduit port.
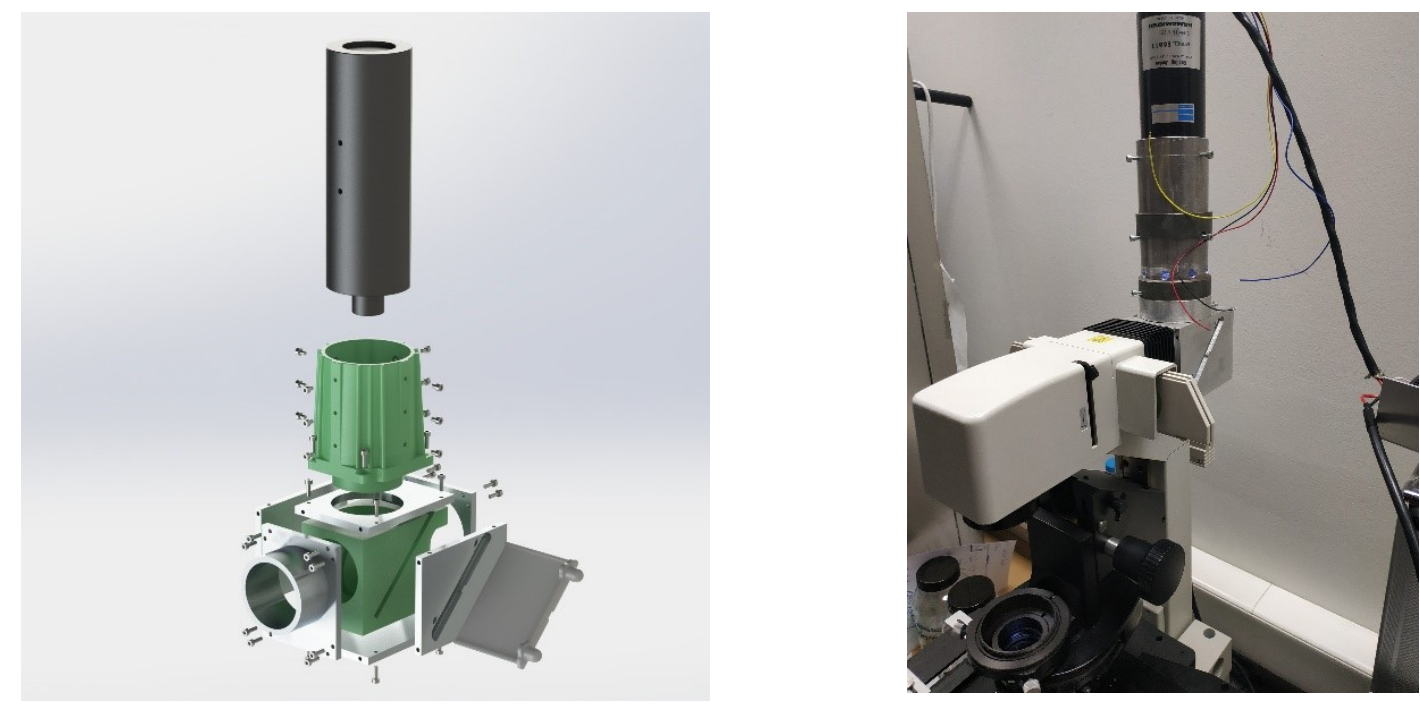

Figure 4. On the left panel, the exploded view of the microscope adaptation is shown. Metal parts are bolted together, the green part is a 3D printed plastic insert for the mirror assembly and stroboscope holder, which is represented in this figure in black color. Mirror tray is fully retraceable, allowing the selection of top light source or direct source from the back of the microscope. The right panel shows a photograph of mounted prototype to the microscope.

\section{Conclusion}

There are many different approaches to measuring bending stiffness. They vary in precision, the spatial and temporal precision of the method and amount of invasiveness to the measured specimen. A closer look of the thermal fluctuation measurement method was presented. The precision of the results depends on the image clarity, focus and blur. 
The method of increasing the precision of the measurement was proposed, based on the experiences from authors in the literature and experiences gained by implementing the electro-mechanical system for the specific microscope in the laboratory.

The phase-contrast microscope was upgraded with flash light to enable registration of sharp images of vesicles for experimental determination of the bending stiffness of the giant unilamellar phospholipid vesicles. The mechanical modifications for attaching the additional light source to the microscope without the secondary light port was made and the electronics with corresponding software for controlling the flash discharge in sync with camera was devised. With modified light source, the analysis of fluctuaction modes is increased, resulting in more precise calculations of bending stiffness. The integration time of the camera is specified to be around $3 \mathrm{~ms}$ and can deviate from this value, depending on the frames-per-second settings. Stroboscopic pulse lasts around $2 \mu$ s and is therefore three orders of magnitude shorter. This does not necessary imply the highest recorded frequency will be three orders of magnitude higher, since this high frequency fluctuation modes tend to be lower in amplitude than low frequency ones and they depend on the stiffness of the vesicle and the type of the solution where the vesicles are in.

Conflicts of Interest: The authors declare no conflict of interest.

\section{References}

1. Boal DH. Mechanics of the cell. 2nd ed ed. Cambridge ; New York, Cambridge University Press. 2012.

2. Browicz T. Further observation of motion phenomena on red blood cells in pathological states. Zbl. med. Wissen. 1890; 28: 625-627.

3. Cucina A, Sterpetti AV, Pupelis G, et al. Shear stress induces changes in the morphology and cytoskeleton organisation of arterial endothelial cells. Eur J Vasc Endovascu Surg. 1995; 9: 86-92. DOI: 10.1016/S1078-5884(05)80230-8.

4. Evans E, Leung A. Adhesivity and rigidity of erythrocyte membrane in relation to wheat germ agglutinin binding. J Cell Biol. 1984; 98: 1201-1208. https://doi.org/10.1083/jcb.98.4.1201

5. Fabry B, Maksym GN, Hubmayr RD, et al. Implications of heterogeneous bead behavior on cell mechanical properties measured with magnetic twisting cytometry. Journal of Magnetism and Magnetic Materials. 1999; 194: 120-125. DOI: 10.1016/S0304-8853(98)00564-2.

6. Fošnarič M, Penič S, Iglič A, Bivas I. Thermal Fluctuations of Phospholipid Vesicles Studied by Monte Carlo Simulations. In: Iglič A, Genova J, editors. Advances in Planar Lipid Bilayers and Liposomes. 2013; 17: 331-357.

DOI: 10.1016/B978-0-12-411516-3.00012-7

7. Genova J, Pavlič J. Realization of Marin Mitov's idea for the stroboscopic illumination used in optical microscopy. Bulgarian Journal of Physics. 2012; 39: 65-71.

8. Genova J, Zheliaskova A, Mitov MD. The influence of sucrose on the elasticity of SOPC lipid membrane studied by the analysis of thermally induced shape fluctuations. Colloids adn Surfaces A. 2006; 282-283: 420-422. DOI:

10.1016/j.colsurfa.2005.11.065

9. Genova J, Zheliaskova A, Vitkova V, Mitov MD. Stroboscopic illumination study of the dynamics of fluctuating vesicles. Journal of optoelectronics and advanced materials. 2009; 11: 1222-1225.

10. Jaasma MJ, Jackson WM, Keaveny TM. Measurement and Characterization of Whole-Cell Mechanical Behavior. Ann Biomed Eng. 2006; 34: 748-758. DOI: 10.1007/s10439-006-9081-0.

11. Jubery TZ, Srivastava SK, Dutta P. Dielectrophoretic separation of bioparticles in microdevices: A review: Microfluidics and Miniaturization. Electrophoresis. 2014; 35: 691-713. DOI: 10.1002/elps.201300424.

12. Klanjšček N, Penič S. Stroboskopski sistem za zajem slik fosfolipidnih veziklov za določanje mehanskih lastnosti. Proceedings of the 30th International Electrotechnical and Computer Science Conference ERK 2021 30: 423-426.

13. Lee C-H, Lin W-C, Wang J. All-optical measurements of the bending rigidity of lipid-vesicle membranes across structural phase transitions. Physical Review E. 2001; 64: 020901. DOI: 10.1103/PhysRevE.64.020901

14. Lin DC, Dimitriadis EK, Horkay F. Robust Strategies for Automated AFM Force Curve Analysis-I. Non-adhesive Indentation of Soft, Inhomogeneous Materials. J Biomech Eng. 2007; 129: 430-440. DOI: 10.1115/1.2720924.

15. Maniotis AJ, Chen CS, Ingber DE. Demonstration of mechanical connections between integrins, cytoskeletal filaments, and nucleoplasm that stabilize nuclear structure. Proceedings of the National Academy of Sciences. 1997; 94: 849-854.

DOI: 10.1073/pnas.94.3.849. 
16. Méléard P, Gerbeaud C, Bardusco P, et al. Mechanical properties of model membranes studied from shape transformations of giant vesicles. Biochimie. 1998; 80: 401-413. DOI: 10.1016/S0300-9084(00)80008-5.

17. Menger FM, Keiper JS. Chemistry and physics of giant vesicles as biomembrane models. Curr Opin Chem Biol. 1998; 2: 726-732. DOI: 10.1016/S1367-5931(98)80110-5.

18. Milner ST, Safran SA. Dynamical fluctuations of droplet microemulsions and vesicles. Physical Review A. 1987; 36: 4371-4379. DOI: 10.1103/PhysRevA.36.4371.

19. Mohandas N, Chasis JA, Shohet SB. The influence of membrane skeleton on red cell deformability, membrane material properties, and shape. Semin Hematology. 1983; 20: 225-242.

20. Morshed A, Karawdeniya BI, Bandara YMNDY, et al. Mechanical characterization of vesicles and cells: A review. Electrophoresis. 2020; 41: 449-470. DOI: 10.1002/elps.201900362.

21. Nussenzveig HM. Cell membrane biophysics with optical tweezers. Eur Biophys J. 2018; 47: 499-514. DOI: $10.1007 / \mathrm{s} 00249-017-1268-9$.

22. Pavalko FM, Chen NX, Turner CH, et al. Fluid shear-induced mechanical signaling in MC3T3-E1 osteoblasts requires cytoskeleton-integrin interactions. American Journal of Physiology-Cell Physiology. 1998; 275:1591-1601. DOI: 10.1152/ajpcell.1998.275.6.C1591.

23. Penič S, Iglič A, Bivas I, et al. Bending elasticity of vesicle membranes studied by Monte Carlo simulations of vesicle thermal shape fluctuations. Soft matter. 2015; 11: 5004-5009. DOI:10.1039/C5SM00431D

24. Phillips R. Physical biology of the cell. Second edition ed. London : New York, NY, Garland Science. 2013.

25. Piontek MC, Lira RB, Roos WH. Active probing of the mechanical properties of biological and synthetic vesicles. Biochim Biophys Acta Gen Subj. 2021; 1865: 129486. DOI: 10.1016/j.bbagen.2019.129486.

26. Schneider MB, Jenkins JT, Webb WW. Thermal fluctuations of large quasi-spherical bimolecular phospholipid vesicles. J. Phys.France 1984; 45: 1457-1472. DOI: 10.1051/jphys:019840045090145700.

27. Shao JY, Hochmuth RM. Micropipette suction for measuring piconewton forces of adhesion and tether formation from neutrophil membranes. Biophys J. 1996; 71: 2892-2901. DOI: 10.1016/S0006-3495(96)79486-9.

28. Song J, Waugh RE. Bending rigidity of SOPC membranes containing cholesterol. Biophys J. 1993; 64: 1967-1970. DOI: 10.1016/S0006-3495(93)81566-2

29. Zhou Y, Raphael RM. Effect of salicylate on the elasticity, bending stiffness, and strength of SOPC membranes. Biophys J. 2005; 89: 1789-1801. DOI: 10.1529/biophysj.104.054510 